\title{
Can Vaginal Washing Fluid Urea, Creatinine and Qualitative $\beta$-hCG Diagnose Suspected Premature Rupture of Membranes?
}

\author{
Omneya M. Osman', Mohamed Elghazaly² \\ ${ }^{1}$ Faculty of Medicine, Kasr Al Ainy Hospital, Cairo University, Cairo, Egypt \\ ${ }^{2}$ Cairo University, Cairo, Egypt \\ Email: omna03@gmail.com, omna03@hotmail.com
}

Received 19 September 2014; revised 15 October 2014; accepted 6 November 2014

Academic Editor: Moon Whan Im, Inha University School of Medicine, Korea

Copyright (C) 2014 by authors and Scientific Research Publishing Inc.

This work is licensed under the Creative Commons Attribution International License (CC BY).

http://creativecommons.org/licenses/by/4.0/

c) (i) Open Access

\section{Abstract}

Several markers have been studied to diagnose PROM but there is still limitation with a gold standard test for the diagnosis [1]. Objective: To evaluate and compare the accuracy of urea, creatinine and hCG in vaginal fluid washing for diagnosis of PROM and to determine cut off levels. Type of the study: Prospective study performed on pregnant women in their third trimester who are coming to Kasr El Ainy maternity outpatient clinic with history of fluid leakage. Patients and methods: 150 patients between 26 - 40 weeks were included in the study. Group I included 50 patients with clinically confirmed ROM, group II included 50 with unconfirmed ROM and 50 controls with no complaints (group III). All patients were subjected to speculum examination for amniotic fluid pooling, nitrazine paper test, vaginal fluid washing for measurement of creatinine, urea and $\beta$-hCG, and ultrasound examination. The results were compared as regards the 3 study groups. Results: There was no statistically significant difference among the 3 study groups as regards age, parity BMI, nor gestational age. There was a statistically significant difference in creatinine level among confirmed, suspected and control groups. Urea levels showed also a statistically significant difference among the three groups. There was a positive correlation between vaginal creatinine and urea and gestational age in the 3 groups $(r=0.43$, and 0.51 respectively). There was a statistically significant difference between vaginal fluid wash urea and creatinine levels and $\beta$-hCG as sensitivity, specificity, positive and negative predictive values and accuracy for urea and creatinine were all $100 \%$ and for qualitative $\beta$-hCG $83 \%, 100 \%, 100 \%, 85.6 \%$, and $91 \%$ respectively. Conclusion: Urea and creatinine in vaginal washings can be accurately used in diagnosing suspected PROM and are more accurate than $\beta$-hCG. 


\section{Keywords}

\section{PROM, Vaginal Fluid Creatinine, Vaginal Fluid Urea, Vaginal Fluid hCG}

\section{Introduction}

Several markers have been studied to diagnose PROM but there is still limitation with a gold standard test for the diagnosis [1].

Premature rupture of membranes (PROM) is defined as rupture of membranes before 37 weeks of pregnancy. It complicates $2 \%$ to $4 \%$ of all singleton and $7 \%$ to $20 \%$ of twin pregnancies. It is the leading cause of preterm labor and accounts for $18 \%$ to $20 \%$ of perinatal deaths and $21.4 \%$ of morbidity [2].

Diagnosis of PROM is easy when the rupture is obvious but difficult and indeed impossible when the rupture is minimal. Therefore any biochemical test used to establish a correct diagnosis must be reliable, simple and rapid [3].

Correct diagnosis of PROM has a great importance because failure of diagnosis can lead to unwanted obstetric complication like chorioamnionitis and preterm birth. On the other hand, over diagnosis can lead to unnecessary intervention like hospitalization and induction of labor [4].

History is reliable in $10 \%$ to $50 \%$ of cases [4]. Although inspection of fluid leakage from the cervix was the traditional method to diagnose PROM, it is associated with $12 \%-30 \%$ false negative results [5]. Nitrazine test can also show false positive or negative results due to contamination by urine (alkaline), blood or meconium, vaginitis, cervicitis or antibiotic use. Ferning test has also 5\% - 30\% false positive and 13\% - 30\% false negative [3] [4]. It should be performed on a sample collected from the posterior fornix or lateral vaginal sidewall to avoid cervical mucus, which may also give a false positive result [6] [7].

Alternative markers have been studied, including alpha-fetoprotein (AFP), fetal fibronectin, insulin-like growth binding protein 1(ILFBP-1), prolactin, beta-subunit of human chorionic gonadotropin ( $\beta$-hCG), creatinine, urea, lactate and placental macroglobulin 1(PAMG-1) [8]. However, despite the improved diagnostic potential of these markers, they have not become popular due to their complexity and high cost [4].

Urea and creatinine may be helpful in diagnosing PROM because fetal urine is the most important source of amniotic fluid (AF) in the second half of pregnancy. Creatinine concentration in AF increase gradually between 20 and 32 weeks of gestation and more rapidly thereafter, when they were two to four times higher than maternal serum [6]. In addition, $\beta$-hCG has been evaluated as a possible predictor of preterm labor and as a marker for PROM [9].

The aim of our study was to evaluate and compare the accuracy of urea, creatinine and hCG in vaginal fluid washing for diagnosis of PROM and to determine cut off levels.

\section{Patients and Methods}

Our study is a prospective study performed on 150 pregnant women in their third trimester (28 - 40 weeks) who were coming to Kasr El Ainy maternity outpatient clinic, 100 complaining of fluid leakage and 50 normal uncomplicated pregnancies. Exclusion criteria were twins, congenital anomalies, vaginal bleeding (spontaneous or traumatic), meconium, presence of uterine contractions, placenta previa, patients with medical complication indicating termination of pregnancy. An informed consent was taken for all patients after the ethics committee of Kasr Al Ainy Hospital agreed to conduct the study.

All patients were subjected to full history taking, general and abdominal examination and sterile Cusco speculum examination. A cotton tip applicator was inserted in posterior vaginal fornix and immediately transferred on nitrazine paper. Alkaline $\mathrm{pH}$ changed yellow paper to blue. Patients who had positive pooling and nitrazine paper test were considered confirmed PROM (group I). Patients with pooling negative and/or nitrazine paper test negative were considered as suspected PROM (group II).

Samples for urea, creatinine and hCG were taken from the posterior fornix which was irrigated with 5cc saline using a sterile syringe then aspirated using the same syringe and sent to the lab immediately for:

1) Measuring creatinine and urea: $3 \mathrm{ml}$ were quantitatively tested for creatinine assay (Ektachem Clinical Chemistry slides, Johnson and Johnson). Urea analysis was done using a spectrometer. 
2) $\beta$-hCG: After shaking the syringe, 2 drops of the collected sample were applied on QUPID plus one step pregnancy (Tabio laboratory Boerne, Texas, USA).

Trans abdominal ultrasound for gestational age, AFI, placental site and congenital anomalies was then performed.

\section{Statistical Analysis}

Was done using statistical program for social science version 12 (SPSS).

Description of quantitative variables was presented as mean, standard deviation (SD) and range, qualitative data as numbers and percentage. Chi-square test was used to compare qualitative variables between groups. Unpaired t-test was used to compare 2 groups as regard quantitative variable in parametric data. Mann Whitney Wilcoxon test was used to compare two groups as regard non-parametric data.

ROC (receiver operator characteristic) curve was used to find out the overall predictivity of urea and creatinine and to find out the best cut off value. $p$ value $<0.05$ was considered significant.

\section{Results}

The demographic data for each group is represented in Table 1.

There was no statistically significant difference between the 3 groups as regards patient age, parity, and BMI and gestational group $(\mathrm{p}>0.05)$.

There was a statistically significant difference in creatinine level between the 3 groups (Table 1 ). With a cut-off level of $>0.23 \mathrm{mg} / \mathrm{dl}$ (ROC curve) for detection of creatinine, the sensitivity, specificity, PPV, NPV and accuracy were all 100\% (Table 2).

There was a statistically significant difference in urea level between confirmed, suspected and control groups (Table 1). The cut off level for detection of urea was $>0.41 \mathrm{mg} / \mathrm{dl}$. Sensitivity, specificity, PPV, NPV and accuracy were all $100 \%$ (Table 2). There was an increase in creatinine, and urea levels with increased gestational age) ( $\mathrm{r}=0.43,0.51$ respectively, $\mathrm{p}<0.05)$.

Vaginal fluid wash $\beta$-hCG was positive in $78 \%$ of GI, $14 \%$ of GII and was negative in GIII patients ( $\mathrm{p}<0.05)$ (Table 1).

Patients who had a positive $\beta$-hCG had higher levels of creatinine than patients who had negative $\beta$-hCG. This difference was statistically significant $(\mathrm{p}<0.05)$ (Table 3 ).

The sensitivity, specificity, PPV, NPV and accuracy of vaginal fluid wash $\beta$-hCG were $83 \%, 100 \%, 100 \%$, $85.6 \%$ and $91 \%$ respectively at a cut off level $20 \mathrm{mIU} / \mathrm{ml}$ (Table 2).

Table 1. Demographic data for the studied groups.

\begin{tabular}{ccccc}
\hline & GI (PROM+) & GII (PROM+/-) & GIII (controls) & p value \\
\hline Age (years) & $25.9 \pm 4.9(18-39)$ & $26.4 \pm 5.4(18-39)$ & $26.2 \pm 6.1(18-41)$ & $>0.05$ \\
Parity & $1.8 \pm 1.1(0-5)$ & $2.1 \pm 1.4(0-6)$ & $2.3 \pm 0.9(0-5)$ & $>0.05$ \\
BMI & $27.3 \pm 2.1(20-32)$ & $28.2 \pm 2.3(21-34)$ & $28.2 \pm 2.5(18-35)$ & $>0.05$ \\
GA (weeks) & $32.5 \pm 2.7(26-36 \mathrm{w})$ & $31.7 \pm 2.9(26-37 \mathrm{w})$ & $32.9 \pm 3.4(26-37 \mathrm{w})$ & $>0.05$ \\
AFI $\leq \mathbf{5}$ (\%) & $38 \%$ & $12 \%$ & $2 \%$ & $<\mathbf{0 . 0 5}$ \\
Vaginal fluid creatinine & $1.1 \pm 0.38(0.65-2.1)$ & $0.31 \pm 0.26(0-0.89)$ & $0.09 \pm 0.076(0-0.27)$ & $<\mathbf{0 . 0 5}$ \\
(mg/d) & $1.87 \pm 0.42(0.67-4.2)$ & $0.32 \pm 0.28(0-0.92)$ & $0.11 \pm 0.091(0-0.21)$ & $<\mathbf{0 . 0 5}$ \\
Vaginal fluid urea (mg/dl) & $14 \%$ & $0 \%$ & $<\mathbf{0 . 0 5}$ \\
Vag.fluid hCG positive (\%) & $78 \%$ & & &
\end{tabular}

Table 2. Sensitivity, specificity, PPV, NPV and accuracy of creatinine, urea, $\beta$-hCG and AFI.

\begin{tabular}{ccccccc}
\hline & Cut off & Sensitivity & Specificity & PPV & NPV & 100 \\
Creatinine & $>0.23 \mathrm{mg} / \mathrm{dl}$ & 100 & 100 & 100 & 100 & 100 \\
Urea & $>0.41 \mathrm{mg} / \mathrm{dl}$ & 100 & 83 & 100 & 100 & 100 \\
$\beta$-hCG & $20 \mathrm{mIU} / \mathrm{ml}$ & 33.2 & 97.4 & 96.3 & 85.6 & 42.1 \\
AFI & $\leq 5$ & & & & 0.67 \\
\hline
\end{tabular}


Table 3. Relationship between creatinine level and $\beta$-hCG among GI.

\begin{tabular}{ccc}
\hline & Creatinine $(\mathrm{mg} / \mathrm{dl})$ & \\
\hline$\beta$-hCG & Mean \pm SD $($ Range $)$ & p value \\
Negative & $0.41 \pm 0.32(0-1.85)$ & $<\mathbf{0 . 0 5}$ \\
Positive & $0.99 \pm 0.51(0.17-2.1)$ & \\
\hline
\end{tabular}

We found creatinine to be significantly higher in $\beta$-HCG positive GI patients ( $<<0.05$ ) (Table 3).

AFI was $\leq 5$ in $38 \%$ of GI, $12 \%$ of GII and $2 \%$ in GIII. This difference was statistically significant (Table 1 ).

AFI cut off was $\leq 5$. Sensitivity, specificity, PPV, NPV and accuracy for detection of PROM were 33.2, 97.4, 96.3, 42.1 and 0.67 respectively.

\section{Discussion}

Diagnosis of PROM is easy when the rupture is obvious, but difficult and indeed impossible when the rupture is minimal. Several markers have been studied to diagnose PROM but there is still limitation with a gold standard test for the diagnosis [1]. Therefore, any biochemical test used to establish a correct diagnosis must be reliable, simple and rapid [3].

Regarding urea and creatinine levels in vaginal fluid, there was a statistically significant difference between confirmed, suspected and control groups ( $\mathrm{p}<0.05)$. The mean vaginal fluid creatinine concentration of confirmed group was $1.11 \mathrm{mg} / \mathrm{dl}, 0.31 \mathrm{mg} / \mathrm{dl}$ in the suspected group, which was, and $0.09 \mathrm{mg} / \mathrm{dl}$ in the control group $(\mathrm{p}<0.05)$

Our results were in concordance with Kafali H and Okuzler C, 2007 [7] who also studied 3 groups and showed a statistically significant difference among all groups regarding creatinine levels. They found the sensitivity, specificity, PPV, NPV and accuracy of creatinine all to be all $100 \%$ at a cut off level $0.6 \mathrm{mg} / \mathrm{dl}$. We had the same results but at a cut off level of $0.23 \mathrm{mg} / \mathrm{dl}$. Gurbuz et al., 2004 [6] studied only 2 groups (54 cases and 34 controls). A cut-off value of $0.12 \mathrm{mg} / \mathrm{dl}$ was proposed for creatinine and sensitivity, specificity, PPV, NPV and accuracy were all $100 \%$. They concluded that vaginal fluid creatinine was cheaper and faster than other methods to establish accurate diagnosis.

Li and Chang, 2000 [10] also studied 2 groups (10 cases and 10 controls). They compared between cases of PROM and controls as regards creatinine, $\beta$-hCG and AFP. The median levels of creatinine in vaginal fluid were $0.95 \mathrm{mg} / \mathrm{dl}$ in cases and 0.05 in controls $(\mathrm{p}=0.0001)$. The sensitivity, specificity, PPV, NPV and accuracy of creatinine were $90 \%, 100 \%, 100 \%, 90.9 \%$ and $95 \%$ respectively. Creatinine was more accurate than the other tests.

Kariman et al., 2010 [11] also studied 2 groups to compare vaginal fluid urea and creatinine in confirmed cases and normal controls. They found the mean level of vaginal fluid urea and creatinine in the PROM group to be significantly higher than in the control group. Kariman et al., 2013 [12] studied 60 confirmed PROM patients, 66 suspected and 53 controls. They concluded that creatinine had higher sensitivity and specificity than urea and that it could be the gold standard for diagnosing PROM.

Creatinine values increase with increased gestational age due to glomerular filtration and maturation of tubular function. A concentration of $1.75 \mathrm{mg} / \mathrm{dl}$ or more correlates with a gestational age of 37 weeks or more.

Similar to Olivera et al. 2000 [13] we also observed an increase in creatinine levels with increased gestational age.

We found the mean vaginal fluid urea concentration of confirmed group to be $1.87 \mathrm{mg} / \mathrm{dl}$, which was higher than the suspected group $(0.32 \mathrm{mg} / \mathrm{dl})$, and the control group $(0.11 \mathrm{mg} / \mathrm{dl})(\mathrm{p}<0.05)$. The sensitivity, specificity, PPV, NPV and accuracy of urea were all $100 \%$ with a cut off value of $0.41 \mathrm{mg} / \mathrm{dl}$.

Kafali and Okuzler, 2007 [7] found the sensitivity, specificity, PPV, NPV and accuracy of urea to be $100 \%$ but at a cut off level of $12 \mathrm{mg} / \mathrm{dl}$. The difference in the cut-off levels between the different studies may be attributed to the different number of patients included, the different inclusion criteria and the different gestational age of studied patients.

As a normal pregnancy progresses, the mean level of $\beta$-hCG in maternal blood increases to approximately $54,000 \mathrm{mIU} / \mathrm{ml}$ at $8-12$ weeks of gestation. It then declines rapidly to around $12,000 \mathrm{mIU} / \mathrm{ml}$ and remains at this level up to the end of the pregnancy. Beta hCG is also present in amniotic fluid at concentrations ranging from $2000-70,000 \mathrm{mIU} / \mathrm{ml}$. In addition, it is secreted by cervical glands and is present in the vaginal secretions 
[14] [15].

Vaginal fluid wash $\beta$-hCG was positive in $78 \%$ of GI, $14 \%$ of GII and $0 \%$ of GIII ( $\mathrm{p}<0.05)$. The sensitivity, specificity, PPV, NPV and accuracy of $\beta$-hCG in detecting PROM were $83 \%, 100 \%, 100 \%, 85.6 \%$ and $91 \%$ respectively (using the bedside urine pregnancy test, cut-off $20 \mathrm{mIU} / \mathrm{ml}$ ). Similar results were obtained by Cooper et al., 2004 [15]. Kim et al., 2005 [4] found the cut-off level of $\beta$-hCG to be $39.8 \mathrm{mIU} / \mathrm{ml}$ with a sensitivity, specificity, PPV, NPV 95.5\%, 94.7\%, 91.3\% and 97.3\%. Other studies also showed higher cut off levels as they used the ROC curve analysis while we used the normal bedside urine pregnancy test kit [3] [7] [16] .

There was a statistically significant difference between the studied groups as regards the AFI. AFI was $\leq 5$ in $38 \%$ of GI, in $12 \%$ of GII and in $2 \%$ of GIII ( $p<0.05)$.

The sensitivity, specificity, PPV, NPV and accuracy of AFI in detecting PROM were 33.2\%, 97.3\%, 96.3\%, $42.1 \%$, and $0.67 \%$ respectively. Kafali and Oksuzler, 2007 [7] concluded that there was no statistically significant difference among the three groups regarding AFI.

We used speculum examination, Nitrazine test, AFI and vaginal fluid urea, creatinine and $\beta$-hCG for diagnosis of PROM and we studied 3 groups of patients and compared them.

We used strict exclusion criteria (twins, congenital anomalies, vaginal bleeding meconium, presence of uterine contractions, placenta previa, patients with medical complication). Weaknesses included not comparing those tests with Amnisure (approved by the WHO) and the use of the bedside pregnancy test as the study was self-funded.

\section{Conclusion}

Our study showed that vaginal fluid washing urea and creatinine levels in vaginal fluid washing were very accurate, simple tests for diagnosis of PROM. Furthermore they were more accurate than $\beta$-hCG.

\section{Disclosure}

There was no conflict of interest.

The study was self funded.

\section{References}

[1] Caughy, A.B., Robinson, J.N. and Norwitz, E.R. (2008) Contemporary Diagnosis and Management of Preterm Premature Rupture of Membranes. Reviews in Obstetrics \& Gynecology, 1, 11-22.

[2] ACOG Committee on Practice Bulletins-Obstetrics (2007) ACOG Practice Bulletin No. 80: Premature Rupture of Membranes. Premature Rupture of Membranes. Clinical Management Guidelines for Obstetricians Gynecologists. Obstetrics \& Gynecology, 109, 1007-1020. http://dx.doi.org/10.1097/01.AOG.0000263888.69178.1f

[3] Esim, E., Turan, C., Unal, O., et al. (2003) Diagnosis of Premature Rupture of Membranes by Identification of $\beta$-HCG in Vaginal Washing Fluid. European Journal of Obstetrics and Gynecology and Reproductive Biology, 107, 37-40. http://dx.doi.org/10.1016/S0301-2115(02)00277-4

[4] Kim, Y.H., Park, Y.W., Kwon, H.S., et al. (2005) Vaginal Fluid $\beta$-Human Chorionic Gonadotropin Level in the Diagnosis of Premature Rupture of Membranes. Acta Obstetricia et Gynecologica Scandinavica, 84, 802-805.

[5] Sucak, A., Moroy, P. and Cakmakl, P. (2005) Insulin-Like Growth Factor Binding Protein-1: A Rapid Detection of Amniotic Fluid Leakage after Amniocentesis. Turkish Journal of Medical Sciences, 35, 157-161.

[6] Gurbuz, A., Karateke, A. and Kabaca, C. (2004) Vaginal Fluid Creatinine in Premature Rupture of Membranes. International Journal of Gynecology \& Obstetrics, 86, 370-371.

[7] Kafali, H. and Okuzler, C. (2007) Vaginal Fluid Urea and Creatinine in Diagnosis of Premature Rupture of Membranes. Archives of Gynecology and Obstetrics, 275, 157-160. http://dx.doi.org/10.1007/s00404-006-0240-1

[8] Lee, S.E., Park, J.S., Norwitz, E.R., et al. (2007) Measurement of Placental Alpha-Microglobulin-1 in Cervicovaginal Discharge to Diagnose Rupture of Membranes. Obstetrics \& Gynecology, 109, 634-640. http://dx.doi.org/10.1097/01.AOG.0000252706.46734.0a

[9] Bernstein, P.S., Stern, R., Lin, N., et al. (1998) $\beta$-Human Chorionic Gonadotropin in Cervicovaginal Secretions as Predictor of Delivery. American Journal of Obstetrics \& Gynecology, 79, 870-873. http://dx.doi.org/10.1016/S0002-9378(98)70180-2

[10] Li, H.Y. and Chang, T.S. (2000) Vaginal Fluid Creatinine, Human Chorionic Gonadotropins and Alpha-Fetoprotein Levels for Detecting Premature Rupture of Membranes. Zhonghua Yi Xue Za Zhi (Taipei), 63, 686-690. 
[11] Kariman, N., Toloui, H., Azarhoush, R., Alavi Majd, H. and Jan-nesari, S.H. (2010) Diagnostic Values of Urea and Creatinine Values of Cervicovaginal Discharges in Determining of Premature Rupture of Membranes. Pajouhesh Dar Pezeshki, 33, 222-227.

[12] Kariman, N., Afrakhte, M., Hedayati, M., Fallahian, M. and Majd, H. (2013) Diagnosis of Premature Rupture of Membranes by Assessment of Urea and Creatinine in Vaginal Washing Fluid. Iran Journal of Reproductive Medicine, 11, 93-100.

[13] Olivera, F.R., Barros, E.G. and Magalhaes, J.A. (2000) Biochemical Profile of Amniotic Fluid for the Assessment of Fetal Renal Development. Brazilian Journal of Medical and Biological Research, 35, 215-222. http://dx.doi.org/10.1590/S0100-879X2002000200010

[14] Movahed, F., Elmizadeh, K., Javadi, A. and Choopani, A. (2012) The Value of Qualitative Detection of Human Chorionic Gonadotropin in Vaginal Washing Fluid for Diagnosis of Preterm Premature Rupture of Membranes. Pakistan Journal of Medical Sciences, 28, 900-903.

[15] Cooper, A.L., Vermillion, S.T. and Soper, D.E. (2004) Qualitative Human Chorionic Gonadotropin Testing of Cervicovaginal Washings for the Detection of Preterm Premature Rupture of Membranes. American Journal of Obstetrics \& Gynecology, 191, 593-597. http://dx.doi.org/10.1016/j.ajog.2004.06.076

[16] Al-Bayati, M., Al-Kazaly, E. and Athrab, S. (2011) Vaginal Washing Fluid $\beta$-HCG Levels for Detecting Premature Rupture of Membranes. The Iraqi Postgraduate Medical Journal, 10, 61-66. 
Scientific Research Publishing (SCIRP) is one of the largest Open Access journal publishers. It is currently publishing more than 200 open access, online, peer-reviewed journals covering a wide range of academic disciplines. SCIRP serves the worldwide academic communities and contributes to the progress and application of science with its publication.

Other selected journals from SCIRP are listed as below. Submit your manuscript to us via either submit@scirp.org or Online Submission Portal.
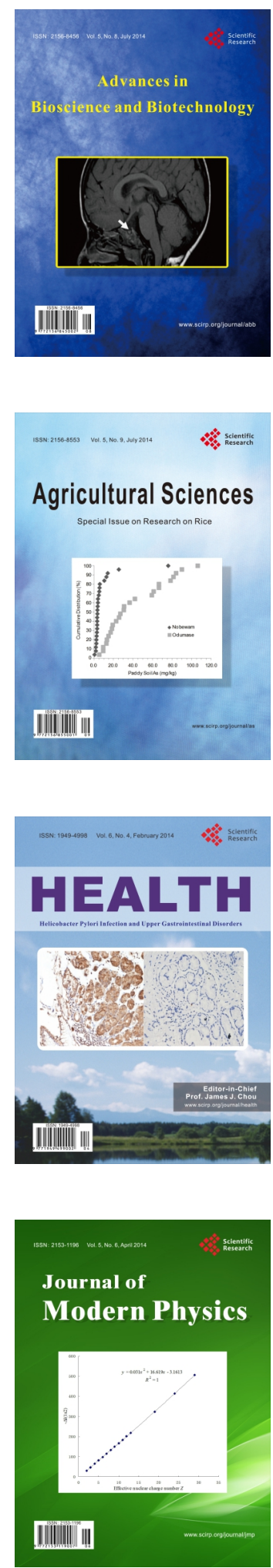
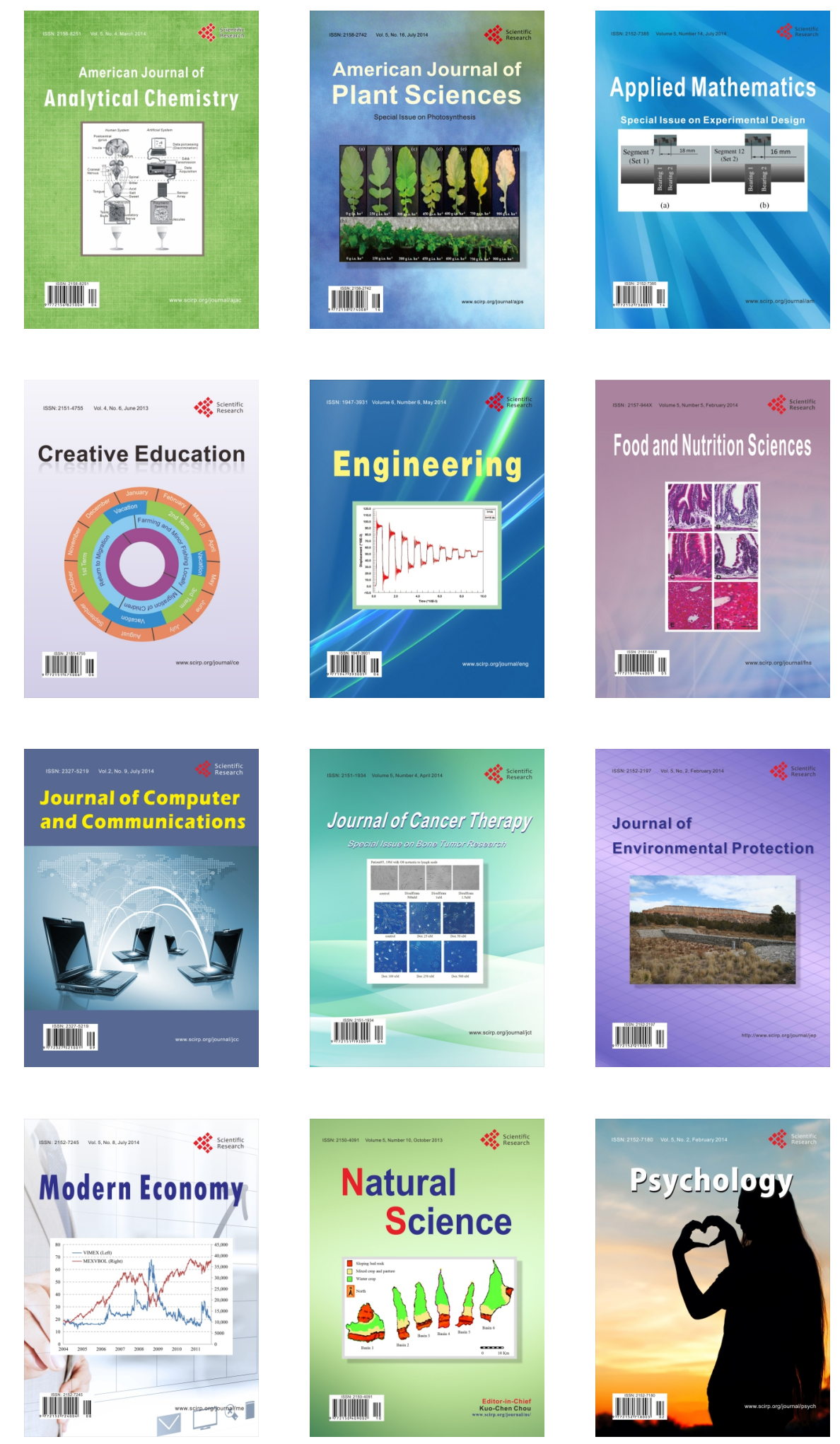